\title{
BMJ Open Study protocol for the ACT response pilot intervention: development, implementation and evaluation of a systems-based Agitation Code Team (ACT) in the emergency department
}

Ambrose H Wong (D) , ${ }^{1}$ Jessica M Ray, ${ }^{1}$ Marc A Auerbach, ${ }^{2}$ Arjun K Venkatesh, ${ }^{1}$ Caitlin McVaney, ${ }^{1}$ Danielle Burness, ${ }^{3}$ Christopher Chmura, ${ }^{3}$ Thomas Saxa, ${ }^{3}$ Mark Sevilla, ${ }^{3}$ Colin T Flood, ${ }^{1}$ Amitkumar Patel, ${ }^{3}$ Travis Whitfill, ${ }^{1}$ James D Dziura, ${ }^{1,4}$ Kimberly A Yonkers, ${ }^{5,6}$ Andrew Ulrich, ${ }^{1}$ Steven L Bernstein ${ }^{1,5}$

To cite: Wong AH, Ray JM, Auerbach MA, et al. Study protocol for the ACT response pilot intervention: development, implementation and evaluation of a systemsbased Agitation Code Team (ACT) in the emergency department. BMJ Open 2020;10:e036982. doi:10.1136/ bmjopen-2020-036982

- Prepublication history for this paper is available online. To view these files, please visit the journal online (http://dx.doi org/10.1136/bmjopen-2020036982).

Received 14 January 2020 Revised 02 March 2020 Accepted 12 May 2020

Check for updates

(C) Author(s) (or their employer(s)) 2020. Re-use permitted under CC BY-NC. No commercial re-use. See rights and permissions. Published by BMJ.

For numbered affiliations see end of article.

Correspondence to

Dr Ambrose H Wong;

wongambrose@gmail.com

\section{ABSTRACT}

Introduction Emergency department (ED) visits for behavioural conditions are rising, with 1.7 million associated episodes of patient agitation occurring annually in acute care settings. When de-escalation techniques fail during agitation management, patients are subject to use of physical restraints and sedatives, which are associated with up to $37 \%$ risk of hypotension, apnoea and physical injuries. At the same time, ED staff report workplace violence due to physical assaults during agitation events. We recently developed a theoretical framework to characterise ED agitation, which identified teamwork as a critical component to reduce harm. Currently, no structured team response protocol for ED agitation addressing both patient and staff safety exists.

Methods and analysis Our proposed study aims to develop and implement the agitation code team (ACT) response intervention, which will consist of a standardised, structured process with defined health worker roles/responsibilities, work processes and clinical protocols. First, we will develop the ACT response intervention in a two-step design loop; conceptual design will engage users in the creation of the prototype, and iterative refinement will occur through in situ simulated agitated patient encounters in the ED to assess and improve the design. Next, we will pilot the intervention in the clinical environment and use a controlled interrupted time series design to evaluate its effect on our primary outcome of patient restraint use. The intervention will be considered efficacious if we effectively lower the rate of restraint use over a 6 -month period.

Ethics and dissemination Ethical approval by the Yale University Human Investigation Committee was obtained in 2019 (HIC \#2000025113). Results will be disseminated through peer-reviewed publications and presentations at scientific meetings for each phase of the study. If this pilot is successful, we plan to formally integrate the ACT response intervention into clinical workflows at all EDs within our entire health system.

\section{INTRODUCTION}

Emergency department (ED) visits for behavioural conditions are common and
Strengths and limitations of this study

- This work will use a human factors systems ap proach to develop a pragmatic, structured teambased protocol with defined health worker roles/ responsibilities and work processes.

- Our approach to investigating agitation and workplace violence in the emergency department simultaneously addresses and balances patient and staff safety.

- We will use quantifiable outcomes of safety for patients and healthcare workers to demonstrate measurable decreases in restraint/sedative use and frequency of assaults as a result of our intervention.

- We will employ a controlled interrupted times series design to evaluate our intervention since randomisation is not possible in this single site study, which may be subject to geopolitical or institutional factors unique to our clinical environment.

- Variable compliance and adoption of the new protocol may be a potential limitation, which we will mitigate through administrative buy-in and use of staff champions.

rising rapidly, with one in in eight visits related to mental health and substance use disorders. ${ }^{1}$ The most severe of these visits are associated with symptoms of acute agitation, which manifest as excessive psychomotor activity leading to violent and disruptive behaviour in patients. ${ }^{2}$ Agitation events occur 1.7 million times a year in emergency settings. ${ }^{3}$ Treatment and management of these acute agitation episodes are complex and pose potential safety threats for both patients themselves and ED staff caring for them. Although physical restraints are commonly used in the ED setting, physical trauma, respiratory depression, and asphyxiation leading 
to cardiac arrest are well-documented risks of restraint placement. ${ }^{4-6}$ Serious adverse effects can also result from sedative use, including a systematic review reporting up to $37 \%$ of cases complicated by respiratory compromise, QT prolongation, paradoxical increase in agitation, and death. ${ }^{7}$ Concurrently, staff are increasingly subject to workplace violence during agitation management, with the ED identified as one of the highest risk healthcare environments. ${ }^{8}$ A recent meta-analysis identified a pooled incidence of 36 workplace violence events for every $10,000 \mathrm{ED}$ patients. ${ }^{9}$ One in five healthcare professionals experienced workplace physical violence perpetrated by patients or visitors worldwide annually. ${ }^{10}$ Studies have consistently demonstrated missed workdays and burnout from exposure to violent incidents. ${ }^{11}$

Expert consensus panels have derived recommendations regarding the management of agitation and prevention of workplace violence in the ED. ${ }^{3}$ However, limited work exists to translate these recommendations into pragmatic interventions that consider both patient and health worker safety. ${ }^{8}$ Using a rigorous, systematic approach to address this critical issue, our team recently proposed a novel framework for ED agitation management based on mixed-methods analysis of staff data. ${ }^{12}$ Our framework identified factors and potential targets for intervention coalescing at five interrelated levels of care delivery: patient, staff, healthcare team, unit environment, and health system. Most importantly, we found that workplace violence and agitation management needed to be considered concurrently as integrated concepts to balance patient safety with prevention of assaults on staff for any potential interventions to be effective. ${ }^{13}$

Structured and coordinated teamwork has long been established as valuable to improving patient safety, with significant reductions in morbidity and clinical errors. ${ }^{14}$ However, a standardised team approach has not been traditionally used in emergency psychiatry, despite the elevated risk to physical safety. ${ }^{16}{ }^{16}$ Healthcare facilities commonly purchase proprietary programmes for agitation management, including the Nonviolent Crisis Intervention used at our institution, ${ }^{17}$ which focus on training for one-on-one interactions between an individual staff member and the patient. In our previous qualitative studies of ED agitation, we observed that the primary gaps in safety resulted from teamwork challenges that were rarely addressed by these commercial products. ${ }^{12} 18$ De-escalation attempts were often more successful when

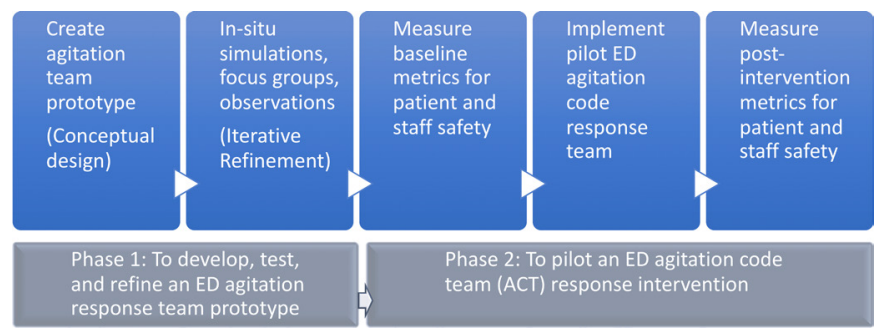

Figure 1 Overview and steps for each phase of ACT response intervention study. ED, emergency department. communication and trust-building occurred between colleagues. On the other hand, professional hierarchy and disparate goals of agitation care created tension and disagreement in treatment plans, leading to increased safety threats. Experts have also recognised this and proposed a structured team approach for ED agitation, comparable to those designed for trauma and cardiac arrest. ${ }^{1619}$ To test this approach, we piloted novel teambased simulations for ED agitation to demonstrate feasibility in a team-based approach to agitation management. ${ }^{20} 21$ Our interprofessional scenarios brought hospital security, ED nurses and emergency physicians together in a simulated clinical encounter. As a result of these simulations, staff participants expressed interest in a team-based protocol for ED agitation and felt that it would improve both patient and staff safety.

Health safety experts are increasingly adopting a human factors systems approach to analyse and redesign healthcare delivery. ${ }^{22}$ This approach considers complex, highpressure industries like healthcare as a set of interrelated elements in a work system, including worker(s), tasks, tools/technologies, physical environment, and organisational conditions to achieve desired safety outcomes. ${ }^{23}$ While this human factors systems approach has frequently been applied to patient safety in the healthcare setting, it has recently been recognised as applicable in examining workplace safety as well. ${ }^{24}$ We were previously successful in applying the guiding principles from this approach to derive our systems-based framework for ED agitation. ${ }^{12}$ Experts in human factors employ user-centred design, direct observations, simulations, and focus groups to improve critical decision making and workflow. ${ }^{25} 26$ These techniques will successfully uncover the complex clinical decisions made during agitation management to create a team-based protocol that maximises safety under fluctuating work conditions and potential physical danger.

\section{Rationale and aims of study}

Our study aims to develop and pilot the Agitation Code Team (ACT) response intervention, which will consist of a standardised, structured process with defined health worker roles/responsibilities, work processes, and clinical protocols. By using a human factors systems approach, the ACT intervention will simultaneously address and balance patient and staff safety in the ED through two aims: (1) to develop the ACT prototype in a two-step loop of conceptual design and iterative refinement and (2) to pilot and evaluate the ACT response intervention for efficacy in the ED.

\section{METHODS AND ANALYSIS Overview}

The ACT response intervention study will occur in two phases (figure 1). Phase I aims to create the ACT response intervention that integrates with clinical workflow and supports health worker task performance, and phase II aims to pilot the ACT response intervention to 


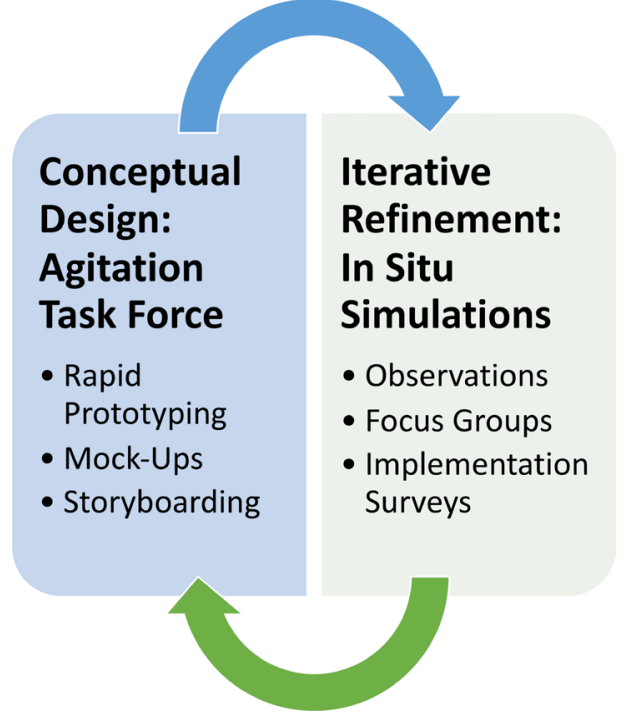

Figure 2 Iterative two-step user-centred design process for development of ACT response prototype.

determine its impact on safety outcomes for patients and staff members in the clinical environment. Results from this investigation will derive a systems-based, data-driven intervention that minimises harm during ED agitation management.

\section{Patient and public involvement}

There were no funds or time allocated for patient and public involvement so we were unable to involve patients. However, we have invited patient advocates to help us develop our dissemination strategy.

\section{Phase I: development and refinement of ACT response prototype}

This phase will occur via an iterative two-step usercentred design ${ }^{27}$ process (figure 2 ) to develop and refine the ACT response prototype: (1) conceptual design to create a model team response structure and protocol, and (2) iterative refinement via in situ simulated agitated patient encounters with a live standardised participant (SP). Through buy-in from hospital stakeholders and clinical staff, we have created a newly-formed interprofessional agitation task force consisting of staff liaisons and representatives from patient safety, social work, legal, emergency psychiatry, protective services and ED leadership. Teamwork factors in agitation management ${ }^{12}$ as well as existing guidelines for managing $\mathrm{ED}$ agitation ${ }^{3}$ will be vetted through the agitation task force to create a model of ACT. Next, in situ simulations will test the developing ACT prototype in the clinical environment. Results from this testing phase will be inputted back into conceptual design for the agitation task force to improve the prototype for further testing in another iterative round of iterative refinement. The process will adapt and improve ACT while assimilating within the ED system until it is ready for clinical implementation.

\section{Conceptual design}

We will first integrate our previously identified teamwork factors ${ }^{12}$ into existing knowledge regarding management of ED agitation to create an initial framework of the ACT response prototype. We will base the constructs for this template (table 1) on results from Project (BETA) Best practices in Evaluation and Treatment of Agitation, ${ }^{3}$ a series of landmark publications describing expert consensus guidelines for best practices in evaluation and treatment of agitation. Next, we will implement conceptual design with the agitation task force to create a formal approach for ACT with a detailed set of roles/responsibilities and clinical protocols. We anticipate that ACT membership will be ad hoc in nature, with individuals from each participating profession fluctuating between different agitation encounters, shifts and times of the day depending on staffing and workflow needs of the ED as a whole. However, defined structures with specified workflow for each individual role of the ACT response will be dictated by the prototype to facilitate team effectiveness. This conceptual design process ${ }^{27}$ will consist of techniques used by leading design thinking firms, including brainstorming, rapid prototype development, physical and graphical mock-ups of the ACT protocol, and storyboarding. ${ }^{28}$ These techniques incorporate user and stakeholder input through early and consistent engagement of the agitation task force in the design process, thus building buy-in and facilitating customisation of the design based on multiple perspectives, needs and preferences. In addition, members of the agitation task force who serve in hospital administrative roles will ensure

Table 1 Proposed constructs for initial template of ACT response prototype

Construct Potential guidelines and measures

Medical Evaluation and Triage Agitation scale(s); laboratory tests and diagnostic imaging; protocols for transfer/ treatment; inclusion/exclusion criteria of patient classes.

Team structure and protocol Personnel and professions to be included; roles/responsibilities of individual members; documentation/electronic health record (EHR) interface; staffing and environmental logistics.

$\begin{array}{ll}\text { Avoidance of restraint and coercion } & \begin{array}{l}\text { Thresholds/indications for use of coercion; types/routes/frequencies/doses of } \\ \text { sedatives; strategies/domains of behavioural techniques/seclusion/de-escalation. }\end{array} \\ \begin{array}{ll}\text { Incorporation of existing relevant } \\ \text { programmes }\end{array} & \begin{array}{l}\text { Staff training; modification of physical space/security; improve/change reporting } \\ \text { mechanisms; worksite analysis; limitations of visitors/patient volume. }\end{array}\end{array}$


integration of ACT into existing workplace safety policies and procedures of our health system that may influence agitation management in the ED.

\section{Iterative refinement}

Once we have created a first draft of the ACT response prototype from the conceptual design phase, we will immediately evaluate the prototype in the clinical environment using simulation techniques. A trained SP acting as an acutely agitated patient will 'arrive' in our ED as an in situ simulated clinical encounter on a biweekly basis to uncover potential systems barriers in usability of the ACT response prototype. Live SPs are frequently employed for in situ simulations that analyse high-stakes human interactions with patients during healthcare delivery. ${ }^{29}{ }^{30}$ Our prior work demonstrated that SPs created a high level of fidelity and facilitated discussion for staff participating in an agitation simulation. ${ }^{1220}$ Staff members on clinical duty will act as participants for the sessions. Each simulation will use variations of a base scenario template developed in our previous work. ${ }^{12}$ The SP will be trained through prescripted prompts and actions, while staying within defined safety measures for the simulation. Immediately following, we will conduct a focus group with the staff participants through structured debriefing for $30 \mathrm{~min}$. Qualitative data will be collected while observing the in situ simulations and from focus groups during simulation debriefings to feed back to the agitation task force for iterative refinement and modification of the prototype after each session. Guest $e t$ al have shown that three to five focus groups uncovered $>90 \%$ of their completed thematic codebooks, respectively. ${ }^{31}$ Thus, we will aim for six to eight simulations held biweekly over a period of 3-4 months. Observations will be made on a qualitative instrument, while debriefings will be guided by open-ended questions relevant to systems elements that affected the simulated encounter. Key constructs of the data collection will reflect elements in the human factors systems approach (table 2$)^{23}$ to assist in seamless integration of the team intervention. Participants in the simulations will also fill out a quantitative survey that asks respondents to rate implementation outcomes for each construct of the ACT response prototype (team, tasks, tools/technology, environment, organisation/process) on a seven-point Likert scale. For each construct, we will also include an open text box field to solicit one area of improvement. We will focus on feasibility as our primary area of interest but will also solicit feedback for the seven areas of implementation outcomes as described by Proctor $e t a l^{32}$ for mental health interventions.

\section{Phase II: pilot implementation and evaluation of ACT response intervention}

This phase will be a prospective quantitative study to measure the effect of the ACT response intervention in reducing restraint use. ${ }^{33}$ Given site-wide implementation of our ACT response protocol and the ad hoc nature of team membership in emergency care, randomisation by individual patients or staff members will not be feasible in this study. Thus, we will use a controlled interrupted time series (CITS) design, which is considered one of the strongest quasi-experimental designs when randomisation is not possible. ${ }^{34}$ Its rigour is derived from its full use of the longitudinal nature of available data and account for preintervention trends through collection at multiple and equally spaced time points before and after an intervention. ${ }^{35}$ Baseline measurements for safety outcomes will be collected from the electronic health record for a 12-month period before implementation of the ACT intervention. We will budget a transition period of 1 month for the intervention to successfully be adopted into established clinical practice. After implementation, these measurements will be repeated for another 12 months for an internal comparison of safety outcomes. Concurrently, these measurements will also be collected at our community site ED, situated fifteen minutes away, with stable measured rates of agitation events. ${ }^{36}$ The community site will not receive the ACT response intervention during the study period, thus serving as the control for the interrupted time series analysis.

Table 2 Observations and focus group data collection constructs

\begin{tabular}{ll} 
Construct & Items \\
\hline $\begin{array}{l}\text { Person (patient) } \\
\text { Team (staff) }\end{array}$ & $\begin{array}{l}\text { Chief complaint(s), type(s)/nature of aggression, level of agitation. } \\
\text { patterns and interactions between team members, roles/responsibilities and list of actions for all } \\
\text { personnel }\end{array}$ \\
\hline Tasks & $\begin{array}{l}\text { Transfer process between prehospital stretcher and patient bed, type/nature of de-escalation } \\
\text { attempts, administration of restraints/sedatives, end outcome(s) of response, patient } \\
\text { reassessment. }\end{array}$ \\
\hline Tools/technology & $\begin{array}{l}\text { Sedative choice/route, type(s) of restraint(s) used, type(s) of stretcher, electronic health record } \\
\text { interface. }\end{array}$ \\
\hline Physical environment & Time of day, associated visitors/patients, safety threat(s) to staff/patient, ED location of response. \\
Organisational conditions & Interface with prehospital services, engagement with law enforcement, patient disposition. \\
\hline
\end{tabular}

ED, emergency department. 


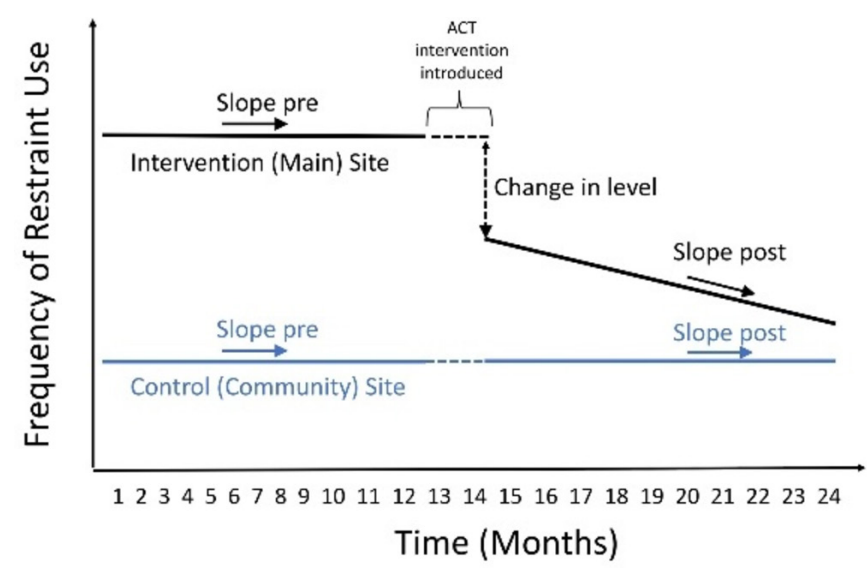

Figure 3 Controlled interrupted time series design. ACT, Agitation Code Team.

\section{Outcomes}

As our primary outcome, we will report biweekly and overall rates of physical restraint use for the predefined 12-month periods preintervention/postintervention at our ED (intervention site) and community affiliate ED (control site) as our metric for patient safety. As secondary outcomes, we will report biweekly and overall rates of sedative use and staff injuries for the 12-month periods preintervention/postintervention at both sites. For the primary analysis, we will use a CITS analysis ${ }^{33}{ }^{37}$ (figure 3) with biweekly measurements of physical restraint/sedative use that occur at the intervention site and control site over the 12-month periods preintervention/postintervention. Using R statistical software (V.3.4.1), we will employ a segmented regression approach using a Poisson Regression (appropriate for modelling rates), assessing the mean restraint use per behavioural health visit and modelling the residuals preintervention and postintervention. Separate models will be constructed for intervention and control sites. Differences in means and slopes between intervention and control groups at baseline, preintervention and postintervention will be included in the segmented regression to adjust for the control. ${ }^{38}$ Autocorrelation will be assessed using the Breusch-Godfrey test, ${ }^{39}$ and adjusted for if needed. The CITS analysis will be supported by $\chi^{2}$ tests between the two periods to determine differences in rates between the preintervention/ postintervention phases for the primary outcome. All analyses will involve two-tailed tests of significance and $p$ values $<0.05$ will be considered statistically significant. The intervention will be considered efficacious if we effectively lower the rate of physical restraint/sedative use at the intervention site.

In our preliminary studies, we were able to observe 95 restraint events over a 2-month period with RAs stationed in the ED for 30 hours a week in randomised 6 hours blocks, which approximates to 570 restraints in 12 months. It has been suggested that a minimum of 12 data points before intervention and 12 after intervention is sufficient for an CITS design. ${ }^{40}$ We aim to have double the number of data points using biweekly time points for
12 months before and 12 months after the intervention. Thus, we expect to have sufficient data for an CITS, and plan to see a total of 1140 events (570 in the each of the preintervention and postintervention phases).

\section{DISCUSSION}

Agitation events in the ED lead to both patient and staff safety threats, and there is a pressing need to develop innovative interventions that will have meaningful impact on measurable safety outcomes. Healthcare sector experts have increasingly adopted a human factors systems approach to address safety for patients. ${ }^{22}$ Our study's innovation lies in its use of a human factors systems approach to improve workflow during agitation management. This approach deliberately places the person(s) in the centre of the system and emphasises that design should focus on users to optimise work performance. This will ensure that our intervention design supports rather than hinders the work required of individuals on the ACT response intervention. Demonstrating feasibility in this type of approach to address workplace violence will provide evidence for using similar methods of systems integration and implementation of workplace safety interventions at other clinical sites.

In addition, our approach to investigating ED agitation simultaneously addresses and balances patient and staff safety. Our prior work identified a care paradox for ED staff during agitation management, where their altruistic desires and sense of professional duty for this marginalised population clash with self-preservation instincts and fear of being harmed, magnifying their stress load and leading to frustration and burnout. ${ }^{18}$ This paradox has not yet been well described, and in fact, the discordance is reflected in the current literature on emergency psychiatry. Separate areas of research exist for staff exposure to workplace violence ${ }^{4142}$ and agitation management for patients focused on safety profiles of sedatives and restraints, ${ }^{7344}$ and it is unclear how interventions benefiting the safety of one group may impact the other. Several case reports have shown feasibility and anecdotal improvement in staff feelings of safety after implementation of a structured code team response to manage agitation, but the evidence is currently limited by lack of preintervention data and unclear impact on patient-based outcomes. ${ }^{45}{ }^{46}$ Our ACT response intervention will be one of the first attempts to address patient safety and staff injuries together as one and the same problem, considering healthcare workers and patients together simultaneously as person(s) in a work system with structured measurements for both staff and patient outcomes. ${ }^{23}$

However, our study contains some limitations. In phase I, we plan to implement in situ simulations and focus groups in the clinical environment to detect and improve the ACT response prototype, which may be superseded by needs of clinical care and workflow. To mitigate this, we will embed the refinement process within our existing monthly in situ simulation programme at our ED, which 
has $>90 \%$ completion of sessions over the past 2 years. With support from the administrative leadership team, we will also schedule additional staff members on simulation days in case of unanticipated rises in volume or acuity. The development process also lacks direct patient input, which may limit the intervention's improvement on patient experience. Our agitation task force will include our grievance coordinator and patient relations staff to ensure advocacy for patients' rights and autonomy in the ACT response design. In addition, we may encounter potential barriers to implementation of the ACT response intervention in Phase II. A 1-month adjustment period has been budgeted into the timeline to allow for adaptation of the new workflow. In addition, we have developed the study in partnership with administrative leadership team to align with the hospital's strategic goals and ensure staff compliance. Staff champions part of the agitation task force will also serve as early adopters in the culture change process. Finally, our single-site design and lack of randomisation may be limited by geopolitical or institutional factors unique to our clinical environment. Our control site will allow for comparisons of relative trends in planned outcomes of interest to overcome this potential limitation.

In summary, our study approaches agitation management and healthcare workplace violence, both critical and rising issues in the $\mathrm{ED}$, using a human factors systems approach to balance patient and staff safety simultaneously, where no clear standard of care exists and evidence is limited. We hope that a structured ACT response intervention will be successfully embedded within existing work processes to effectively balance staff and patient safety. We initiated the conceptual design step of phase I and plan to complete development by June 2020 in time for pilot implementation in the summer of 2020.

\section{ETHICS AND DISSEMINATION}

Ethical approval by the Yale University Human Investigation Committee was obtained in 2019 (HIC\#2000025113). All staff participants will provide informed verbal consent prior to being enrolled in the study, and a waiver of consent for patient subjects has been granted. Results will be disseminated through peer-reviewed publications and presentations at international scientific meetings for each phase of the study. If this pilot is successful, we plan to formally integrate the ACT response intervention into processes and clinical workflows at all EDs within our entire health system, which includes eight emergency care sites across the state of Connecticut, USA.

\footnotetext{
Author affiliations

${ }^{1}$ Department of Emergency Medicine, Yale University, New Haven, Connecticut, USA ${ }^{2}$ Department of Pediatrics and Emergency Medicine, Yale University, New Haven, Connecticut, USA

${ }^{3}$ Department of Emergency Medicine, Yale-New Haven Hospital, New Haven, Connecticut, USA

${ }^{4}$ Department of Biostatistics, Yale School of Public Health, New Haven, CT, United States
}

${ }^{5}$ Department of Chronic Disease Epidemiology, Yale School of Public Health, New Haven, CT, United States

${ }^{6}$ Departments of Psychiatry and Obstetrics \& Gynecology, Yale University, New Haven, Connecticut, USA

Twitter Ambrose H Wong @ambrosehwong and Arjun K Venkatesh @ arjunvenkatesh

Contributors AHW, MAA, JMR, CM, TW and SLB designed the study protocol and obtained funding. AHW is responsible for the overall logistical and scientific aspects of the study, data collection and analysis, and draft of this manuscript. AU, AKV, CC, $\mathrm{DB}, \mathrm{TS}$ and MS provided administrative and logistical support for the study. CTF, AP, JDD and KAY assisted with biostatistical and analytic support for the study design and proposed methods for intervention evaluation. All authors contributed to critical revisions and gave final approval of the manuscript.

Funding This study was supported by the Robert E. Leet and Clara Guthrie Patterson Trust Mentored Research Award and the KL2 TR001862 from the National Center for Advancing Translational Science, components of the National Institutes of Health and the National Institutes of Health Roadmap for Medical Research.

Competing interests None declared.

Patient and public involvement Patients and/or the public were not involved in the design, or conduct, or reporting, or dissemination plans of this research.

Patient consent for publication Not required.

Provenance and peer review Not commissioned; externally peer reviewed.

Open access This is an open access article distributed in accordance with the Creative Commons Attribution Non Commercial (CC BY-NC 4.0) license, which permits others to distribute, remix, adapt, build upon this work non-commercially, and license their derivative works on different terms, provided the original work is properly cited, appropriate credit is given, any changes made indicated, and the use is non-commercial. See: http://creativecommons.org/licenses/by-nc/4.0/.

ORCID iD

Ambrose H Wong http://orcid.org/0000-0001-7471-1647

\section{REFERENCES}

1 Capp R, Hardy R, Lindrooth R, et al. National trends in emergency department visits by adults with mental health disorders. J Emerg Med 2016;51:131-5.

2 Nordstrom K, Zun LS, Wilson MP, et al. Medical evaluation and triage of the agitated patient: consensus statement of the American association for emergency psychiatry project beta medical evaluation workgroup. West J Emerg Med 2012;13:3-10.

3 Holloman GH, Zeller SL. Overview of project beta: best practices in evaluation and treatment of agitation. West $J$ Emerg Med 2012;13:1-2.

4 Mohr WK, Petti TA, Mohr BD. Adverse effects associated with physical restraint. Can J Psychiatry 2003;48:330-7.

5 Zun LS. A prospective study of the complication rate of use of patient restraint in the emergency department. $J$ Emerg Med 2003;24:119-24.

6 Karger B, Fracasso T, Pfeiffer H. Fatalities related to medical restraint devices-asphyxia is a common finding. Forensic Sci Int 2008;178:178-84.

7 Korczak V, Kirby A, Gunja N. Chemical agents for the sedation of agitated patients in the ED: a systematic review. Am J Emerg Med 2016;34:2426-31.

8 Phillips JP. Workplace violence against health care workers in the United States. N Engl J Med 2016;374:1661-9.

9 Nikathil S, Olaussen A, Gocentas RA, et al. Review article: workplace violence in the emergency department: a systematic review and meta analysis. Emerg Med Australas 2017;29:265-75.

10 Li Y-L, Li R-Q, Qiu D, et al. Prevalence of workplace physical violence against health care professionals by patients and visitors: a systematic review and meta-analysis. Int J Environ Res Public Health 2020;17. doi:10.3390/ijerph17010299. [Epub ahead of print: 01 Jan 2020].

11 Taylor JL, Rew L. A systematic review of the literature: workplace violence in the emergency department. J Clin Nurs 2011;20:1072-85.

12 Wong AH, Ruppel H, Crispino LJ, et al. Deriving a framework for a systems approach to agitated patient care in the emergency department. Jt Comm J Qual Patient Saf 2018;44:279-92. 
13 Wong AH, Ray JM, lennaco JD. Workplace violence in health care and agitation management: safety for patients and health care professionals are two sides of the same coin. Jt Comm J Qual Patient Saf 2019;45:71-3.

14 Weaver SJ, Dy SM, Rosen MA. Team-training in healthcare: a narrative synthesis of the literature. BMJ Qual Saf 2014;23:359-72.

15 Manser T. Teamwork and patient safety in dynamic domains of healthcare: a review of the literature. Acta Anaesthesiol Scand 2009;53:143-51.

16 Rintoul Y, Wynaden D, McGowan S. Managing aggression in the emergency department: promoting an interdisciplinary approach. Int Emerg Nurs 2009;17:122-7.

17 Morrison EF. An evaluation of four programs for the management of aggression in psychiatric settings. Arch Psychiatr Nurs 2003;17:146-55.

18 Wong AH-W, Combellick J, Wispelwey BA, et al. The patient care paradox: an interprofessional qualitative study of agitated patient care in the emergency department. Acad Emerg Med 2017;24:226-35.

19 Gillespie GL, Gates DM, Miller M, et al. Emergency department workers' perceptions of security officers' effectiveness during violent events. Work 2012;42:21-7.

20 Wong $\mathrm{AH}$, Wing $\mathrm{L}$, Weiss $\mathrm{B}$, et al. Coordinating a team response to behavioral emergencies in the emergency department: a simulationenhanced interprofessional curriculum. West J Emerg Med 2015;16:859-65.

21 Wong $\mathrm{AH}$, Auerbach MA, Ruppel $\mathrm{H}$, et al. Addressing dual patient and staff safety through a team-based standardized patient simulation for agitation management in the emergency department. Simul Healthc 2018;13:154-62.

22 Pronovost PJ, Goeschel CA, Marsteller JA, et al. Framework for patient safety research and improvement. Circulation 2009;119:330-7.

23 Holden RJ, Carayon P, Gurses AP, et al. SEIPS 2.0: a human factors framework for studying and improving the work of healthcare professionals and patients. Ergonomics 2013;56:1669-86.

24 Carayon P, Hancock P, Leveson N, et al. Advancing a sociotechnical systems approach to workplace safety--developing the conceptual framework. Ergonomics 2015;58:548-64.

25 Militello LG, Hutton RJ. Applied cognitive task analysis (ACTA): a practitioner's toolkit for understanding cognitive task demands. Ergonomics 1998;41:1618-41.

26 Hayden EM, Wong AH, Ackerman J, et al. Human factors and simulation in emergency medicine. Acad Emerg Med 2018;25:221-9.

27 Ray JM, Ahmed OM, Solad Y, et al. Computerized clinical decision support system for emergency department-initiated buprenorphine for opioid use disorder: user-centered design. JMIR Hum Factors 2019;6:e13121.

28 Roberts JP, Fisher TR, Trowbridge MJ, et al. A design thinking framework for healthcare management and innovation. Healthc 2016;4:11-14.

29 Hamman WR, Beaudin-Seiler BM, Beaubien JM, et al. Using in situ simulation to identify and resolve latent environmental threats to patient safety: case study involving operational changes in a labor and delivery ward. Qual Manag Health Care 2010;19:226-30.

30 Medwid K, Smith S, Gang M. Use of in-situ simulation to investigate latent safety threats prior to opening a new emergency department. Saf Sci 2015;77:19-24.

31 Guest G, Namey E, McKenna K. How many focus groups are enough? building an evidence base for Nonprobability sample sizes. Field methods 2017;29:3-22.

32 Proctor E, Silmere H, Raghavan R, et al. Outcomes for implementation research: conceptual distinctions, measurement challenges, and research agenda. Adm Policy Ment Health 2011;38:65-76.

33 Lopez Bernal J, Cummins S, Gasparrini A. The use of controls in interrupted time series studies of public health interventions. Int $\mathrm{J}$ Epidemiol 2018;47:2082-93.

34 Kontopantelis E, Doran T, Springate DA, et al. Regression based quasi-experimental approach when randomisation is not an option: interrupted time series analysis. BMJ 2015;350:h2750.

35 Bernal JL, Cummins S, Gasparrini A. Interrupted time series regression for the evaluation of public health interventions: a tutorial. Int J Epidemiol 2017;46:348-55.

36 Wong AH, Taylor RA, Ray JM, et al. Physical restraint use in adult patients presenting to a general emergency department. Ann Emerg Med 2019;73:183-92.

37 Taljaard M, McKenzie JE, Ramsay CR, et al. The use of segmented regression in analysing interrupted time series studies: an example in pre-hospital ambulance care. Implement Sci 2014;9:77.

38 Linden A, Adams JL. Applying a propensity score-based weighting model to interrupted time series data: improving causal inference in programme evaluation. J Eval Clin Pract 2011;17:1231-8.

39 Godfrey LG. Misspecification tests and their uses in econometrics. J Stat Plan Inference 1996;49:241-60.

40 Wagner AK, Soumerai SB, Zhang F, et al. Segmented regression analysis of interrupted time series studies in medication use research. J Clin Pharm Ther 2002;27:299-309.

41 Behnam M, Tillotson RD, Davis SM, et al. Violence in the emergency department: a national survey of emergency medicine residents and attending physicians. J Emerg Med 2011;40:565-79.

42 Stowell KR, Hughes NP, Rozel JS. Violence in the emergency department. Psychiatr Clin North Am 2016;39:557-66.

43 Riddell J, Tran A, Bengiamin R, et al. Ketamine as a first-line treatment for severely agitated emergency department patients. Am $J$ Emerg Med 2017;35:1000-4.

44 Downey LVA, Zun LS, Gonzales SJ. Frequency of alternative to restraints and seclusion and uses of agitation reduction techniques in the emergency department. Gen Hosp Psychiatry 2007;29:470-4.

45 Downes MA, Healy P, Page CB, et al. Structured team approach to the agitated patient in the emergency department. Emerg Med Australas 2009;21:196-202.

46 Kelley EC. Reducing violence in the emergency department: a rapid response team approach. J Emerg Nurs 2014;40:60-4. 